\title{
Propionibacterium spp. in prosthetic joint infections: a diagnostic challenge
}

\author{
Björn Zappe $\cdot$ Susanne Graf $\cdot$ Peter E. Ochsner $\cdot$ \\ Werner Zimmerli · Parham Sendi
}

Received: 21 May 2007 / Published online: 15 September 2007

(C) Springer-Verlag 2007

\begin{abstract}
Introduction Propionibacterium species are common inhabitants of the skin and usually non-pathogenic for humans. However, Propionibacterium spp. can occasionally cause infections, but are estimated to play a minor role in prosthetic joint infections (PJI). The relative frequency of these anaerobes and their potential to cause surgical site infection may be clinically underestimated. An unknown proportion of these infections might be missed, since little is known about their clinical presentation, and since growth of Propionibacterium spp. in diagnostic samples is often interpreted as contamination. Thus, a hypothesis is being tested, stating that Propionibacterium spp. is not as rare as often reported, and it can cause severe soft-tissue damages in PJI.

Materials and methods In this retrospective analysis, we reviewed all PJI that had been treated in our institution from 2000 to 2005, and assessed the relative frequency of those caused by Propionibacterium spp. In the identified cases, features that led to the diagnosis (clinical, laboratory, radiological, microbiological and histopathological characteristics) were analysed.
\end{abstract}

No funding was available.

B. Zappe $\cdot$ P. E. Ochsner

Clinic of Orthopaedic Surgery, Kantonsspital, Liestal,

Switzerland

W. Zimmerli · P. Sendi $(\bowtie)$

Unit of Infectious Diseases,

Basel University Medical Clinic Liestal,

Rheinstrasse 26, 4410 Liestal, Switzerland

e-mail: sendi-pa@magnet.ch

S. Graf

Microbiology Laboratory, Kantonsspital, Liestal, Switzerland
Results Of 139 cases of prosthetic joint infections, 8(6\%) were caused by Propionibacterium spp. Seven patients complained of pain as the main symptom, and four had damaged soft-tissue. Analysis of the diagnostic procedures showed a median of $39 \%$ positive samples out of all cultured biopsies (median 9.5 biopsies per case), with a median time-to-positivity of 8 days. Results of histopathological examinations of the periprosthetic tissue correlated well with the clinical courses.

Conclusions Our data suggest that Propionibacterium associated prosthetic joint infections occur at a relative frequency that is comparable to many other pathogens. Clinical signs are generally subtle, but the spectrum includes also significant soft-tissue damages. In this study, a median of 9.5 biopsies per case, an incubation time of 14 days, and the aid of histopathological examinations proved to be helpful in establishing the diagnosis.

Keywords Prosthesis-related infections .

Propionibacterium - Anaerobic bacteria $\cdot$ Replacement arthropasties $\cdot$ Postoperative complications

\section{Introduction}

Propionibacterium species are Gram-positive, non-spore forming, anaerobic rods and common inhabitants of the skin; they are usually considered as non-pathogenic for humans. However, these microorganisms can occasionally cause severe infections, including endocarditis, pneumonia, brain abscess, arthritis, osteomyelitis and prosthetic joint associated infections (PJI) $[3,4,6,10,12,14,19,31]$. These microorganisms are estimated to play a minor role in PJI, being isolated in only $2-4 \%$ of the cases $[16,20]$. However, an unknown proportion of Propionibacterium associated PJI 
may not be detected due to several reasons. First, isolation of these anaerobes by standard culture techniques is difficult, due to their microbiological properties. Second, belonging to the skin flora, their growth in cultures of diagnostic specimens is often interpreted as contamination [4]. Third, in contrast to other pathogens, such as Staphylococcus aureus, little is known about the spectrum of clinical presentation of Propionibacterium associated PJI; this leads to doubts whether these anaerobes, normally causing low-grade infections, can by their own cause severe soft-tissue damages. Therefore, diagnosing Propionibacterium associated PJI represents a challenge in clinical practice.

However, based on our own observation, we raised the hypothesis that Propionibacterium spp. is not as rare as often reported, and indeed, can cause severe soft-tissue damages in PJI. The reason for that observation may be due to the use of a rational diagnostic concept that is applied in our centre, including multiple sampling and histopathological examinations. Thereby, a ratio of "culture-positive biopsies to totally taken biopsies" and the comparison with the clinical course, microbiological and histopathological results can help to distinguish between contaminants and causative pathogens.

In this study, we analysed retrospectively Propionibacterium associated PJI that had been treated in our institution from 2000 to 2005, assessed their relative frequency and reviewed the clinical, radiological, microbiological and histopathological characteristics that led to the diagnosis.

\section{Materials and methods}

\section{Diagnosis of PJI}

In addition to clinical signs, such as pain, effusion, erythema, induration, edema at the implant site or sinus tract communicating with the prosthesis, the growth of the same microorganism in at least two cultures of synovia and/or periprosthetic tissue was required [2]. In case of a relapse of PJI with the same species, one positive sample was sufficient.

\section{Study population}

The cases were identified among a cohort of 139 patients with PJI that had been treated in our institution from January 2000 to December 2005. This number corresponds to $39 \%$ of all revision arthroplasties (355) performed in our clinic during this time period; $65 \%$ of these 139 PJI were referred cases. Our clinic for orthopedic surgery is a 48-bed unit acting as a primary care centre for all types of orthopedic surgery of the extremities, as well as a tertiary care centre for patients needing revision arthroplasty.

\section{Case definition}

Patients were included in the study, if the above-mentioned criteria for PJI were fulfilled, and a Propionibacterium spp. was identified as the causative pathogen. In the case of polymicrobial infection, the same criteria were required for each single species.

\section{Informed consent}

At admission to our centre, all patients were asked whether they were willing to allow the use of their information for research purposes. In this study, consent has been received from all involved patients.

\section{Clinical presentation}

Patient charts were reviewed for signs of PJI in their history prior to proven diagnosis. Clinical signs and symptoms, as well as the time of onset were analysed. The condition of the soft tissue was evaluated and categorised as either intact/slightly damaged or moderately/severely damaged [32]; the latter one included the presence of an abscess or a sinus tract.

Laboratory diagnostics

C-reactive protein (CRP) and white blood cell (WBC) count were evaluated at presentation of clinical symptoms, and prior to any intervention (e.g. joint puncture).

Imaging diagnostics

At the time of diagnosis, plain radiographs of the prosthesis were compared with images taken shortly after implantation. The condition of the implant was examined for signs of loosening and/or periprosthetic osteolysis, and graded as stable or unstable [22, 25]. In case of inconclusive clinical presentation and normal findings in the conventional X-ray, a nuclear scintigraphy was performed [9].

Microbiological diagnostics

Analysis included culture results of synovial fluid (obtained by preoperative joint aspiration) and of periprosthetic tissue.

Synovial fluid was aspirated in an operation room under sterile conditions and transported to the microbiology laboratory within $2 \mathrm{~h}$. Specimens were analysed by Gram stain and then directly inoculated in various culture media (Brain-heart bouillon, Columbia sheep blood, Chocolate and Brucella agar, bioMériux, France). Incubation was performed aerobically and anaerobically (Brucella agar, 
incubator filled with $10 \% \mathrm{H}_{2}, 10 \% \mathrm{CO}_{2}$ and $80 \% \mathrm{~N}_{2}$; residual $\mathrm{O}_{2}$ is removed through the use of a palladium catalyst; incubation temperature $35 \pm 2{ }^{\circ} \mathrm{C}$ ).

According to our in-house practice, at least four biopsies of periprosthetic tissue were obtained from each patient at the site of suspected infection (e.g. macroscopic inflammation). Pre- and intra-operative findings, such as sinus tract or pus, prompted a higher number of samples. Each specimen was collected in a sterile and separate box to avoid cross-contamination, and transported to the microbiology laboratory within $2 \mathrm{~h}$. Then each biopsy was inoculated in a separate tube with liquid medium (brain-heart bouillon, bioMérieux, France), and incubated for 14 days. As soon as growth in the liquid medium was visible, subcultures were performed on agar plates (Columbia sheep blood, Chocolate and Brucella, bioMériux, France). However, routine subculturing on agar plates was performed on days 2 and 10 of the 14-day incubation period.

Bacteria were identified by using all of the following methods: Gram-staining, catalase and agglutination test, as well as the VITEK II system (bioMérieux, France). Propionibacterium spp. in particular was identified by using Gram-staining, catalase and indol tests, as well as the RapID ANA II system (Remel, Lenexa, Kansas State, USA).

In the detected cases, the number of totally taken biopsies, the number of culture-positive biopsies, the timeto-positivity, the ratio of culture-positive to totally taken biopsies, and co-infections with other pathogens were evaluated. Minimal inhibitory concentrations (MICs) were examined using $E$ tests (AB Biodisk, Solna, Sweden) on Muller-Hinton and Muller-Hinton blood agar (bioMériux, France).

\section{Histopathological diagnostics}

Through the following method every microbiological finding could be assigned to a histopathological result. During surgical procedures, each single biopsy was numbered and cut in two parts, one for microbiological, and one for histopathological examination. Thus, identical numbers corresponded to two parts from the same biopsy.

Tissue samples for histopathology were processed and embedded in paraffin. Sections were cut using aseptic techniques, and stained with haematoxylin and eosin. Specimens were analysed for polymorphonuclear leucocytes, lymphocytes and tissue macrophages $[5,15]$. The presence of five or more neutrophils per high-power field at a magnification of 400 was defined as acute inflammation and the presence of lymphocytes, plasma cells and/or macrophages, without neutrophils was defined as chronic inflammation [1]. In addition, sections were Gram-stained in case of suspicion of PJI by the clinician or by the pathologist (acute inflammation in haematoxylin and eosin staining).

\section{Results}

Patients

Of 139 patients, we identified 8 cases with PJI due to Propionibacterium spp. including 4 hip, 3 knee and 1 shoulder prostheses. The cases included one female and seven male patients with a median age of 59.5 (39-82) years. This corresponded to $6 \%$ of the causing agents of all PJI (Table 1). In comparison to other pathogens, only staphylococcal and streptococcal infections were more frequent.

\section{Clinical presentation}

The data on clinical presentation prior to identification of Propionibacterium spp. are presented in Table 2. Onset of symptoms that were likely related to PJI occurred in four patients (50\%) within the first month and in three patients (37.5\%) between 7 and 20 months of implanting the prosthesis. However, in six of these seven patients, diagnosis was significantly delayed, with a median duration of symptoms of 10 months (IQR 4.5-19). In only one of these seven patients (case 6), diagnosis was also established without any delay. In this patient, serosanguinous discharge at the implant site occurred after primary wound healing. PJI was confirmed by surgical revision, shortly thereafter. One out of eight patients complained of symptoms at a very late stage (case 1). Six patients suffered from persistent pain, and one patient (case 1) complained of pain only on movement. None of the patients reported fever or chills. Only one patient (case 5) was treated with antimicrobial agents prior to obtaining specimens for diagnosis (e.g. blood samples, synovial fluid, biopsies). Case 5 received fusidic acid, which was discontinued on the day of admission.

Table 1 Cultured microorganisms in prosthetic joint infection

\begin{tabular}{lrrlrr}
\hline Pathogen/prosthesis involved & Hip & Knee & Shoulder & Total & $\%$ \\
\hline $\begin{array}{l}\text { Coagulase-negative } \\
\quad \text { staphylococci }\end{array}$ & 31 & 22 & 2 & 55 & 39 \\
Staphylococcus aureus & 22 & 7 & - & 29 & 21 \\
Streptococcus spp. & 8 & 2 & - & 10 & 7 \\
Propionibacterium spp. & 4 & 3 & 1 & 8 & 6 \\
Pseudomonas aeruginosa & 4 & - & - & 4 & 3 \\
Enterococcus spp. & - & 4 & - & 4 & 3 \\
Peptostreptococcus spp. & 3 & 1 & - & 4 & 3 \\
Escherichia coli & - & 3 & - & 3 & 2 \\
Others & 10 & 1 & - & 11 & 8 \\
Mixed flora & 3 & 3 & - & 6 & 4 \\
No microorganisms detected & 2 & 2 & 1 & 5 & 4 \\
Total & 87 & 48 & 4 & 139 & 100
\end{tabular}




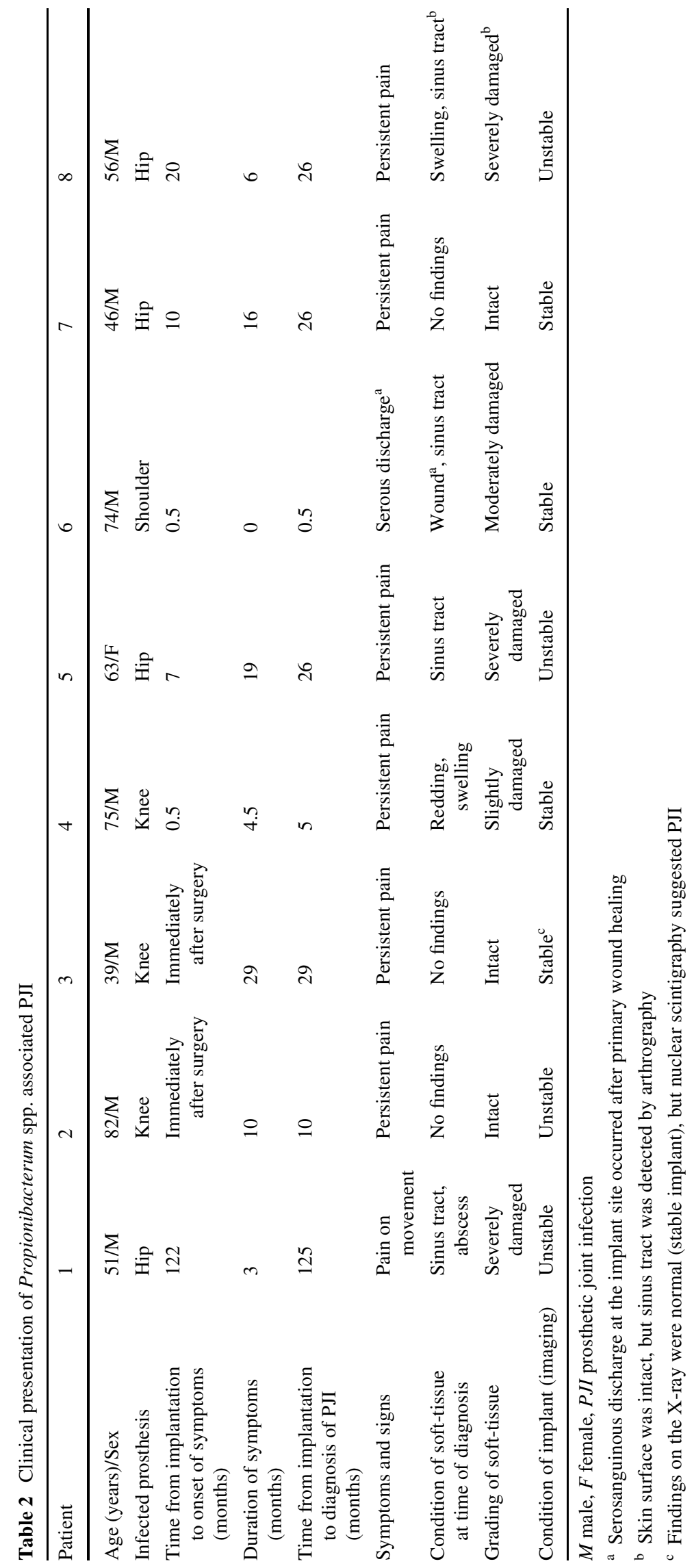


At the time of diagnosis, clinical and radiological examinations (including arthrography) showed a sinus tract and/ or an abscess in four patients; these cases were graded as "moderately/severely" damaged. In one patient, swelling and red discoloration was detected at the implant site, and in three patients no pathological findings of the soft-tissue were found; these were graded as "intact/slightly damaged".

\section{Laboratory diagnostics}

The median CRP value was $17 \mathrm{mg} / \mathrm{l}$ (normal $<5 \mathrm{mg} / \mathrm{l}$ ). In six patients the values ranged from 6 to $20 \mathrm{mg} / \mathrm{l}$, whereas in the other two patients 68 and $75 \mathrm{mg} / \mathrm{l}$ were noted. The median WBC count was 8.1 (IQR 6.2-16.7) $\times 10^{9}$ cells/1 (normal 4-10 $\times 10^{9}$ cells/l).

Imaging studies

Migration of the implant and/or periprosthetic osteolysis was detected by conventional X-ray in four cases. In one patient, findings on the $\mathrm{X}$-ray were normal, but three-phase bone scintigraphy with technetium- 99 m labeled methylene diphosphonate suggested PJI. In two patients imaging was unremarkable, but scintigraphy was not performed, because clinical findings were strongly suggestive of PJI. In one patient with stable implant (case 7), further imaging analysis was not performed, despite inconclusive clinical findings.

\section{Microbiological diagnostics}

Propionibacterium acnes was identified in all but one case (case 5), in which the species was not identified. In four of the cases, synovial fluid was Gram-stained and cultured, but showed no bacteria or growth, respectively. In two of the other cases, PJI was evident from clinical signs, and since a surgical procedure was inevitably required, culturing of synovial fluid was not performed (case 1: fistula, case 6: persistent wound secretion 2 weeks after implantation). In the remaining two patients infection was primarily misdiagnosed as aseptic implant loosening (case 2) or pain due to malpositioning of the implant components (case 7).

The median number of biopsies taken during surgery was 9.5 (IQR 6-10) with a median time-to-positivity of 8 days (IQR 4.5-11) (Table 3). The median percentage of positive cultures from the biopsy samples was $39 \%$ (IQR 17.5-55). In one patient (case 8), Propionibacterium spp. grew in only one out of nine biopsies. This patient was referred to our centre because he was suffering from a relapse of a Propionibacterium spp. associated PJI, and therefore included in the study. In addition, histopathological examination in this case showed signs of acute inflammation in all biopsies. In the first episode of infection, Propionibacterium spp. grew in four of four biopsies and the patient was treated with clindamycin. The MIC for clindamycin was not examined in the first episode; however, it proved to be at a resistance level in the culture specimen from the relapse.

Co-infection was present in two patients (cases 2 and 7), namely with coagulase-negative staphylococci (CoNS) (Table 3). In two other patients (cases 1 and 5), microbiological results showed growth of CoNS in one single specimen, but were interpreted as contaminants, according to the definition criteria for PJI.

MICs were tested for amoxicillin in four cases, for penicillin and ceftriaxone in five cases, and for clindamycin and rifampin in six cases. In two cases, these data were not available. All MICs tested proved to be in the susceptible range, except the one for clindamycin in one case (patient 8).

Histopathological diagnostics

Histopathological examination showed signs of acute inflammation in one case, chronic and acute inflammation in two cases, and signs of chronic inflammation in five cases. In all but two cases (cases 2 and 7) sections were Gram-stained but revealed no bacteria.

\section{Discussion}

The growth of Propionibacterium spp. in cultures of synovial fluid and periprosthetic tissue often raises the question of relevance in PJI. Several reports have shown that these cases represented a diagnostic challenge in clinical orthopedics $[11,23]$. These arguments may influence the estimation that these microorganisms play a minor role in PJI. However, based on our own observation, we raised the hypothesis that Propionibacterium spp. is not as rare as often reported and can cause severe soft-tissue damages in PJI.

The relative frequency of Propionibacterium associated PJI in our retrospective study was higher than in most published series [16, 20]. However, it may be even higher, since our routine microbiological investigations did not include specific media for anaerobic cultures other than Brucella agar (e.g. thioglycolate, fastidious anaerobe broth), and since sonification of removed implants was not performed. Thus, PJI due to Propionibacterium spp. indeed occurred at a comparable rate in PJI than many other microorganisms [8, 21]. In this investigation, only staphylococcal and streptococcal infections occurred more frequently.

Propionibacterium spp. is mainly associated with lowgrade infections, which typically present with subtle signs 
Table 3 Microbiological and histopathological results

\begin{tabular}{|c|c|c|c|c|c|c|c|c|}
\hline Patient & 1 & 2 & 3 & 4 & 5 & 6 & 7 & 8 \\
\hline Age (years)/sex & $51 / \mathrm{M}$ & $82 / \mathrm{M}$ & $39 / \mathrm{M}$ & $75 / \mathrm{M}$ & $63 / \mathrm{F}$ & $74 / \mathrm{M}$ & $46 / \mathrm{M}$ & $56 / \mathrm{M}$ \\
\hline Infected prosthesis & Hip & Knee & Knee & Knee & Hip & Shoulder & Hip & Hip \\
\hline Propionibacterium species & P. acnes & P. acnes & P. acnes & P. acnes & $\begin{array}{l}\text { Not } \\
\quad \text { identified }\end{array}$ & P. acnes & P. acnes & P. acnes \\
\hline Total number of biopsies & 10 & 7 & 10 & 10 & 13 & 4 & 5 & 9 \\
\hline $\begin{array}{l}\text { Number of positive } \\
\text { biopsies with } \\
\text { Propionibacterium spp. } \\
\text { (\% positive) }\end{array}$ & $7(70 \%)$ & $2(29 \%)$ & $4(40 \%)$ & $2(20 \%)$ & $2(15 \%)$ & $2(50 \%)$ & $3(60 \%)$ & $1^{\mathrm{a}}(11 \%)$ \\
\hline $\begin{array}{l}\text { Time-to-positivity } \\
\text { (growth in days) }\end{array}$ & 3 & 12 & 5 & 10 & 14 & 6 & 4 & 10 \\
\hline Co-infection & No & Yes & No & No & No & No & Yes & No \\
\hline $\begin{array}{l}\text { Pathogen (positive/total } \\
\text { biopsies) }\end{array}$ & & CoNS $(2 / 7)$ & & & & & CoNS $(3 / 5)$ & \\
\hline \multicolumn{9}{|l|}{ MIC ( $E$ test; mg/l) } \\
\hline Penicillin & 0.002 & 0.03 & 0.03 & $\begin{array}{l}\text { Not } \\
\quad \text { available }\end{array}$ & 0.015 & $\begin{array}{l}\text { Not } \\
\quad \text { available }\end{array}$ & 0.015 & $\mathrm{nt}$ \\
\hline Amoxicillin & 0.015 & 0.125 & 0.06 & & 0.03 & & $\mathrm{Nt}$ & $\mathrm{Nt}$ \\
\hline Ceftriaxone & 0.015 & 0.125 & 0.125 & & 0.06 & & $\mathrm{Nt}$ & 0.5 \\
\hline Clindamycin & 0.015 & 0.06 & 0.06 & & 0.5 & & 0.03 & $>256$ \\
\hline Rifampin & 0.004 & 0.002 & 0.004 & & 0.004 & & 0.004 & 0.5 \\
\hline $\begin{array}{l}\text { Histopathology } \\
\text { (inflammation) }\end{array}$ & $\begin{array}{l}\text { Acute } \\
\text { and chronic }\end{array}$ & Chronic & Chronic & Chronic & Acute & $\begin{array}{l}\text { Minor } \\
\text { chronic }\end{array}$ & Chronic & $\begin{array}{l}\text { Acute } \\
\text { and chronic }\end{array}$ \\
\hline
\end{tabular}

$N T$ not tested. Gram-staining was performed in all but two patients (case 2 and 7), but showed no bacteria

${ }^{\text {a }}$ This patient was suffering from a relapse of a Propionibacterium spp. associated PJI. In the first episode of infection, Propionibacterium spp. grew in four of four biopsies. The patient was treated with clindamycin, but MIC for clindamycin was at a resistant level (>256 mg/l) in the culture specimen from the relapse

and often occur within 24 months of implantation [32]. In this study, seven out of eight patients complained of pain without more specific symptoms, and the infection symptoms presented in all but one case (case 1) within 24 months of implantation. Most of these low-grade infections may be acquired during implantation and not by haematogenous seeding [13, 17, 32], although this relationship is difficult to demonstrate conclusively. The delay in the diagnosis is often related to the low virulence and slow growth of these microorganisms. Yet, they still can cause severe tissue damage and implant failure as shown in 50\% of our cases. An extensive sinus-tract in a Propionibacterium associated PJI is illustrated in Fig. 1 (case 1). Awareness of this bacterial feature is important, in particular when interpreting culture results in PJI with moderately or severe soft-tissue damages.

Not surprisingly, neither WBC count nor CRP values were helpful in establishing or excluding the diagnosis of Propionibacterium associated PJI.

Preoperative joint puncture should be strived for, irrespective of the pathogen. Culture of synovial fluid (in pediatric blood culture bottles) has been reported to have a

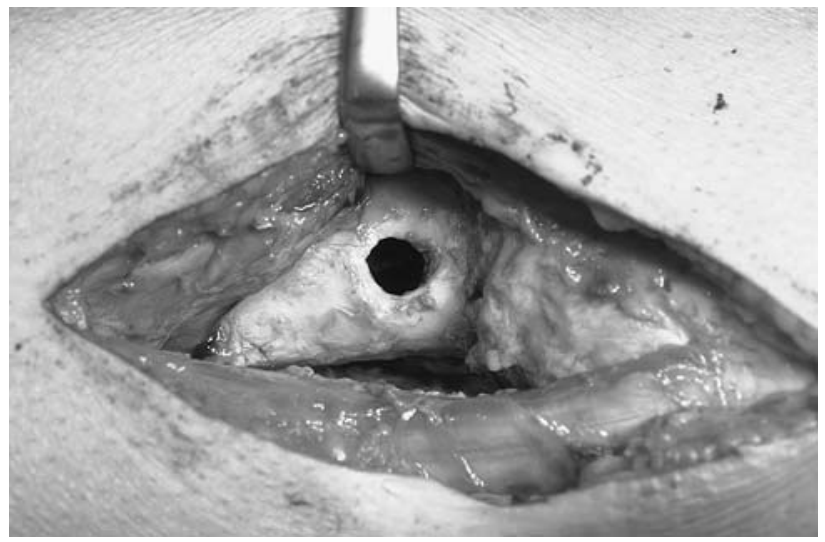

Fig. 1 Case 1: Prosthetic joint infection with severely damaged soft-tissue, including a large fistula through the bone. In seven out of ten obtained biopsies growth of Propionibacterium acnes was detected

sensitivity of $77 \%$ and a specificity of $99 \%$ [26]. In the case of Propionibacterium associated PJI, this procedure may not always be helpful [8]. In four of our cases, synovial fluid was cultured, but revealed no growth. 
Several methods have been suggested to improve the detection rate of these infections, such as a long incubation time $[6,12]$ or the use of sonification of removed implants $[28,29]$. The median time-to-positivity in this series was 8 days, and is comparable to findings of others [12]. However, since these microorganisms grow slowly and need anaerobic conditions, the sensitivity of culture results remains unsatisfactory. To overcome this deficit, several biopsies are obtained. The optimal number of biopsies has not yet been defined. A thoroughly performed prospective study, looking at the detection rate of several microorganisms in periprosthetic tissue specimens, recommended six samples [1]. In our study, a median of 9.5 biopsies per case was taken which may explain the relative frequency of $6 \%$ Propionibacterium spp. associated PJI. Due to the retrospective study-design and the lack of a control group, it remains speculative whether in certain cases fewer biopsies might have missed the diagnosis. Nevertheless, our data and those of Atkins et al. support the concept of culturing six (or even more) specimens, in particular in low grade PJI. Routine culture of removed implants is often associated with contamination and shows a low sensitivity, probably because bacteria are not dislodged from the biofilm [27-29]. Culturing sonicated fluid, though, shows very promising results in improving the diagnosis of infection $[28,29]$. Since in clinical practice this method is not yet widely available, the sensitivity of culture results can be increased by obtaining a higher number of biopsies.

In two of our cases, co-infection with CoNS was detected, which raises difficulties to discriminate between pathogens and contaminants. However, Propionibacterium spp. occurs not rarely in mixed flora [12], and thus likely represent true pathogens in these two cases. Many pathogens grow faster than Propionibacterium spp. and are consequently isolated earlier. Thus, discontinuation of incubation includes the risk of incomplete diagnosis.

Thorough analyses of the involved pathogens, including antimicrobial susceptibility and MIC testing, is important for the medical management of these infections. Propionibacterium spp. are typically susceptible to penicillin, ceftriaxone and clindamycin. However, resistant strains do exist [30], and antimicrobial treatment without previous MIC testing might result in failure, despite correct surgical therapy. In combination with other antimicrobial agents, rifampin is often used in the treatment of PJI, and therefore was tested here also. It has bactericidal activity against surfaceadhering, slow-growing and biofilm-producing staphylococci [33], but to our knowledge its efficacy has not been tested for Propionibacterium spp. residing in biofilms [18]. Yet, previous [7] and our investigations have demonstrated low MIC values that might justify further clinical investigations. Nevertheless, when rifampin is considered, MIC testing should be demanded and, importantly, the compound never be administered alone, since some pathogens rapidly develop resistance [24].

Histopathological examination of the periprosthetic tissue can support infection diagnosis, because the level of inflammation often correlates well with the clinical course $[5,29]$. In our series, four of eight patients had signs of chronic infection, which are compatible with the long duration of symptoms. In three cases signs of acute infection were detected (including the cases with signs of acute and chronic infection), and in all of these, soft-tissue was moderately or severely damaged. In one patient (case 6), infection was detected immediately, which might explain the lack of specific histopathological signs.

In conclusion, isolation of Propionibacterium spp. from PJI should not a priori be interpreted as contamination, but requires further analyses. Our study suggests that these infections occur at a relative frequency that is comparable to many other pathogens (not including staphylococci and streptococci). Most of the cases in our series presented with a long duration of symptoms and subtle signs, but $50 \%$ revealed damaged soft-tissues and unstable implants. The diagnosis was established with a median of 9.5 biopsies per case, an incubation time of 14 days and the aid of histopathological examinations.

Acknowledgments We like to thank Docent Bengt Gårdlund (Department of Infectious Diseases, Karolinska University Hospital Huddinge, Stockholm, Sweden) for his helpful comments. We are grateful to the laboratory technician from our Unit of Microbiology and Anna Bächtold from our library for superb assistance. This study was presented at the 92nd Conference of the German Society of Orthopedics and Orthopedic Surgery, Berlin, Germany, 2-6 October 2006.

Conflict of interest statement None of the authors has a conflict of interest. Ethical Board Review statement: In this retrospective analysis, all data are anonymously presented, and patient consent has been received from all involved humans prior to the study.

\section{References}

1. Atkins BL, Athanasou N, Deeks JJ et al (1998) Prospective evaluation of criteria for microbiological diagnosis of prosthetic-joint infection at revision arthroplasty. The OSIRIS Collaborative Study Group. J Clin Microbiol 36:2932-2939

2. Berbari EF, Hanssen AD, Duffy MC et al (1998) Risk factors for prosthetic joint infection: case-control study. Clin Infect Dis 27:1247-1254

3. Brook I, Frazier EH (1993) Anaerobic osteomyelitis and arthritis in a military hospital: a 10-year experience. Am J Med 94:21-28

4. Brook I, Frazier EH (1991) Infections caused by Propionibacterium species. Rev Infect Dis 13:819-822

5. Fehring TK, McAlister JA Jr (1994) Frozen histologic section as a guide to sepsis in revision joint arthroplasty. Clin Orthop Relat Res 304:229-237

6. Günthard H, Hany A, Turina M et al (1994) Propionibacterium acnes as a cause of aggressive aortic valve endocarditis and 
importance of tissue grinding: case report and review. J Clin Microbiol 32:3043-3045

7. Hoeffler U, Ko HL, Pulverer G (1976) Antimicrobiol susceptibility of Propinibacterium acnes and related microbial species. Antimicrob Agents Chemother 10:387-394

8. Ince A, Seemann K, Frommelt L et al (2004) One-stage revision of shoulder arthroplasty in the case of periprosthetic infection. Z Orthop Ihre Grenzgeb 142:611-617

9. Ivancevic V, Perka C, Hasart O et al (2002) Imaging of low-grade bone infection with a technetium-99 m labelled monoclonal antiNCA-90 Fab' fragment in patients with previous joint surgery. Eur J Nucl Med Mol Imaging 29:547-551

10. Jakab E, Zbinden R, Gubler J et al (1996) Severe infections caused by Propionibacterium acnes: an underestimated pathogen in late postoperative infections. Yale J Biol Med 69:477-482

11. Launder WJ, Hungerford DS (1981) Late infection of total hip arthroplasty with Propionibacterium acnes: a case and review of the literature. Clin Orthop Relat Res 157:170-177

12. Lutz MF, Berthelot P, Fresard A et al (2005) Arthroplastic and osteosynthetic infections due to Propionibacterium acnes: a retrospective study of 52 cases, 1995-2002. Eur J Clin Microbiol Infect Dis 24:739-744

13. Maderazo EG, Judson S, Pasternak H (1988) Late infections of total joint prostheses. A review and recommendations for prevention. Clin Orthop Relat Res 229:131-142

14. Mathisen GE, Meyer RD, George WL et al (1984) Brain abscess and cerebritis. Rev Infect Dis 6(Suppl 1):S101-S106

15. Ochsner PE, Hailemariam S (2006) Histology of osteosynthesis associated bone infection. Injury 37(Suppl 2):S49-S58

16. Pandey R, Berendt AR, Athanasou NA (2000) Histological and microbiological findings in non-infected and infected revision arthroplasty tissues. The OSIRIS Collaborative Study Group. Oxford skeletal infection research and intervention service. Arch Orthop Trauma Surg 120:570-574

17. Poss R, Thornhill TS, Ewald FC et al (1984) Factors influencing the incidence and outcome of infection following total joint arthroplasty. Clin Orthop Relat Res 182:117-126

18. Ramage G, Tunney MM, Patrick S et al (2003) Formation of Propionibacterium acnes biofilms on orthopaedic biomaterials and their susceptibility to antimicrobials. Biomaterials 24:32213227

19. Richards BS (1995) Delayed infections following posterior spinal instrumentation for the treatment of idiopathic scoliosis. J Bone Joint Surg Am 77:524-529
20. Segawa H, Tsukayama DT, Kyle RF et al (1999) Infection after total knee arthroplasty. A retrospective study of the treatment of eighty-one infections. J Bone Joint Surg Am 81:1434-1445

21. Senneville E, Savage C, Nallet I et al (2006) Improved aero-anaerobe recovery from infected prosthetic joint samples taken from 72 patients and collected intraoperatively in Rosenow's broth. Acta Orthop 77:120-124

22. Stumpe KD, Notzli HP, Zanetti M et al (2004) FDG PET for differentiation of infection and aseptic loosening in total hip replacements: comparison with conventional radiography and three-phase bone scintigraphy. Radiology 231:333-341

23. Sulkowski MS, Abolnik IZ, Morris EI et al (1994) Infectious arthritis due to Propionibacterium acnes in a prosthetic joint. Clin Infect Dis 19:224-225

24. Tenover FC (2006) Mechanisms of antimicrobial resistance in bacteria. Am J Med 119:S3-S10; discussion S62-S70

25. Tigges S, Stiles RG, Roberson JR (1994) Appearance of septic hip prostheses on plain radiographs. AJR Am J Roentgenol 163:377380

26. Trampuz A, Hanssen AD, Osmon DR et al (2004) Synovial fluid leukocyte count and differential for the diagnosis of prosthetic knee infection. Am J Med 117:556-562

27. Trampuz A, Piper KE, Hanssen AD et al (2006) Sonication of explanted prosthetic components in bags for diagnosis of prosthetic joint infection is associated with risk of contamination. J Clin Microbiol 44:628-631

28. Trampuz A, Piper KE, Jacobson MJ et al (2007) Sonication of removed hip and knee prostheses for diagnosis of infection. N Engl J Med 357:654-663

29. Tunney MM, Patrick S, Gorman SP et al (1998) Improved detection of infection in hip replacements. A currently underestimated problem. J Bone Joint Surg Br 80:568-572

30. Tyrrell KL, Citron DM, Warren YA et al (2006) In vitro activities of daptomycin, vancomycin, and penicillin against Clostridium difficile, C. perfringens, Finegoldia magna, and Propionibacterium acnes. Antimicrob Agents Chemother 50:2728-2731

31. Yocum RC, McArthur J, Petty BG et al (1982) Septic arthritis caused by Propionibacterium acnes. JAMA 248:1740-1741

32. Zimmerli W, Trampuz A, Ochsner PE (2004) Prosthetic-joint infections. N Engl J Med 351:1645-1654

33. Zimmerli W, Widmer AF, Blatter M et al (1998) Role of rifampin for treatment of orthopedic implant-related staphylococcal infections: a randomized controlled trial. Foreign-Body Infection (FBI) Study Group. JAMA 279:1537-1541 